\title{
Diseño de un programa guía de actividades para la enseñanza de la química en educación media basado en el Modelo Didáctico por Investigación Dirigida
}

\author{
Activities program guide for the teaching of chemistry aimet at students in middle \\ education based in the Didactic Model for Directed Investigation \\ Julie Marcela Rueda Rodríguez ${ }^{1}$, Diana Patricia Hemández Bonilla ${ }^{1}$ \\ y William Femando Castrillón Cardona²
}

${ }^{1}$ Licenciada en química. Universidad Distrital Francisco José de Caldas. ${ }^{2}$ Docente Proyecto Curricular Licenciatura en química. Universidad Distrital Francisco José de Caldas jumarceruro@gmail.com, wfcastrillon@udistrital.edu.co

\section{Resumen}

Se diseño un programa Guía de Actividades para la enseñanza de dos conceptos fundamentales de la química, mezdas y reacciones químicas, dirigido a la educación media. Para este fin, se tuvieron en auenta los estándares curriculares en ciencias naturales de los grados decimo y undécimo, el modelo didáctico de investigación dirigida basándose en la estrategia de resolución de problemas y el uso de algunas tecnologías de información y comunicación - TIC.

Dicho PGA se denominó: "QUIMIVIDA: Cotidianidad y Ciencia", donde se ponen al descubierto algunas de las principales características que se deben tener en cuenta para desarrollar un currículo de aula mucho más aplicado y que tiene en cuenta los modelos didácticos contemporáneos y la cotidianidad de los estudiantes, iniciando así un largo camino en el cual se diseñaran y aplicarán nuevas actividades para los diferentes temas tratados en la educación media, complementando el diseño inicial de dicho PGA.

\section{Palabras clave}

Programa Guía de Actividades, Investigación Dirigida, resolución de problemas, estándares auriaulares en ciencias, competencias básicas en ciencias.

\section{Abstract}

Activities' program Guide (PGA) was designed for the teaching of two fundamental chemistry concepts, mixtures and chemical reactions, aimed at students in middle school. For this purpose, we took into account, the natural science curriculum standards in the tenth and eleventh grades, the model of research-led teaching and problem-solving strategy as well as the use of information and communication technologies - TICs.

"QUIMIVIDA: Science and everyday life," reveals some key characteristics that must be taken into account to develop a curriculum of dassroom and applied over the model takes into account contemporary teaching and everyday life of students

\section{Keywords}

Activities programs guide, problem solving, directed investigation, standard curricular in science, basic natural sciences competitions.

Introducción

El propósito de la presente investigación, es considerar algunos modelos y estrategias estudiados por expertos en didáctica de las ciencias y a partir de ellos, brindar una ayuda a docentes y 
estudiantes de los niveles de media vocacional, mediante la elaboración de un programa guía de actividades (PGA) que favorezca el aprendizaje de la química como un proceso de estructuración de conocimiento cient́fico escolar, soportado en el modelo de enseñanza por investigación dirigida y en una consecuente estrategia de aprendizaje por resolución de problemas.

\section{Contenido}

El Programa Guía de Actividades se diseñó para dos temas de química, mezdas (13 actividades) y reacciones químicas (14 actividades), donde se tuvo en quenta el nivel de complejidad (Tabla 1) de cada actividad para el orden de su presentación, lo que no hace que dichas actividades sean inamovibles, pues el docente es siempre un actor importante del proceso de enseñanza aprendizaje, por lo aual su citerio es el que, finalmente, decide el orden de presentación de dichas actividades diseñadas. De la misma forma, al diseñarse dos módulos diferentes, se logró realizar sugerencias al docente titular, las cuales guian el proceso hacia el cumplimiento de los objetivos de cada actividad.

Así, a continuación se presenta una tabla (Tabla 2) donde se resumen algunas de las actividades que se encuentran en dicho diseño, su complejidad y, la estrategia, modelo o herramienta utilizada, para lograr el fin de este PGA. Dicha complejidad se encuentra en una escala del 1 al 4, en donde 1 es lo menos complejo y 4 el nivel máximo de complejidad. Dicha complejidad se ve representada por la siguiente escala (tabla 1), la cual ha sido propuesta por las autoras del trabajo.

Tabla 1. Escala de Complejidad de las actividades del PGA.

\begin{tabular}{|c|c|c|}
\hline $\begin{array}{l}\text { NIVEL DE } \\
\text { COMPLEJIDAD }\end{array}$ & $\begin{array}{l}\text { COMPETENCIA BÁSICA } \\
\text { DESARROLADA }\end{array}$ & DESCRIPCIÓN \\
\hline 1 & Interpretar & $\begin{array}{l}\text { La interpretación se da en procesos de lectura y de } \\
\text { experimentación. }\end{array}$ \\
\hline 2 & Argumentar & $\begin{array}{l}\text { A argumentar el estudiante debe conocer el tema para así } \\
\text { poder defender su punto de vista con bases fuertes. }\end{array}$ \\
\hline 3 & Proponer & $\begin{array}{l}\text { Al proponer, el estudiante muestra que ha empezado a } \\
\text { comprender claramente los conceptos. }\end{array}$ \\
\hline 4 & Proponer y Argumentar & $\begin{array}{l}\text { Cuando el estudiante propone, y a la vez, tiene los } \\
\text { argumentos suficientes para defender su punto de vista, ha } \\
\text { generado relaciones que le permiten aplicar a diferentes } \\
\text { contextos. }\end{array}$ \\
\hline
\end{tabular}


Tabla 2. Resumen de algunas actividades presentadas en el PGA.

\begin{tabular}{|c|c|c|c|c|}
\hline in & ACTIVIDAD & COMPLEIDAD & $\begin{array}{c}\text { ESTRATE } \\
\text { GIA/HERRAMIENTA/ } \\
\text { MODELO UTILIZADO }\end{array}$ & DESCRIPCIÓN \\
\hline 0 & $\begin{array}{cc}\frac{1}{8} \\
3\end{array}$ & 1 & $\begin{array}{l}\text { Lectura } \\
\text { introductoria }\end{array}$ & $\begin{array}{l}\text { A través de una lectura se busca realizar una introducción } \\
\text { general al tema, mostrando su importancia, en este punto se } \\
\text { busca que el estudiante se apropie de los conocimientos a } \\
\text { través de las preguntas que el mismo puede generar. } \\
\text { Cotidianidad. }\end{array}$ \\
\hline$\frac{2}{2}$ & $\frac{70}{80}$ & 1 & $\begin{array}{l}\text { Resolución de } \\
\text { Problemas } \\
\text { Sopa de letras }\end{array}$ & $\begin{array}{l}\text { Se utiliza la resolución de problemas y se tienen en cuenta las } \\
\text { ideas previas del estudiante, lo que ayuda a enmarcar la } \\
\text { actividad en esta estrategia. Con una sopa de letras se guía la } \\
\text { resolución del problema allí presentado, ¿Todo lo que consumes } \\
\text { está compuesto por una sola sustancia? }\end{array}$ \\
\hline 8 & 옵옹을 & 1 & Mapa conceptual & $\begin{array}{l}\text { El mapa conceptual ayuda a unificar y contrastar las } \\
\text { definiciones de la mayoría de los conceptos encontrados en la } \\
\text { sopa de letras de la actividad anterior. }\end{array}$ \\
\hline$\frac{8}{8}$ & 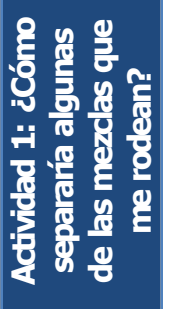 & 2 & $\begin{array}{l}\text { Investigación } \\
\text { dirigida }\end{array}$ & $\begin{array}{l}\text { Se presenta una actividad de apareamiento, donde se pregunta } \\
\text { por mezcla homogénea y heterogénea. Posteriormente se } \\
\text { presenta una serie de mezdas para que el estudiante comience } \\
\text { a observar e identificar los tipos de separación de mezdas. } \\
\text { Al buscar que el estudiante proponga métodos de separación, } \\
\text { se está llegando a obtener pensamiento crítico, lo que lleva a la } \\
\text { investigación, y como se les dan situaciones espećficas se hace } \\
\text { más daro el uso del modelo. }\end{array}$ \\
\hline \multirow{4}{*}{ 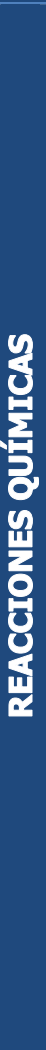 } & 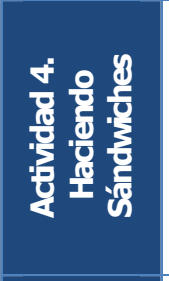 & 3 & $\begin{array}{l}\text { Estrategia } \\
\text { didáctica } \\
\text { (analogía) }\end{array}$ & $\begin{array}{l}\text { Se pide al estudiante prepare cierta cantidad de sándwiches } \\
\text { teniendo en cuenta ciertas características, logrando luego de } \\
\text { una breve explicación establecer la relación con el balanceo y } \\
\text { ecuaciones químicas. } \\
\text { Esta actividad es una estrategia didáctica (analogía) que } \\
\text { permitirá que el estudiante consolide los conocimientos acerca } \\
\text { del balanceo de ecuaciones químicas. }\end{array}$ \\
\hline & 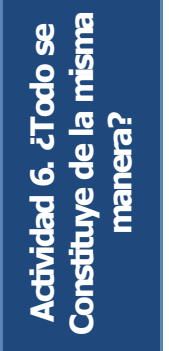 & 4 & $\begin{array}{l}\text { Resolución de } \\
\text { Problemas y } \\
\text { Investigación } \\
\text { dirigida }\end{array}$ & $\begin{array}{l}\text { Se presenta una serie de procedimientos prácticos } \\
\text { experimentales, en donde el estudiante podrá evidenciar los } \\
\text { diferentes tipos de reacciones químicas. Se tiene la opción de } \\
\text { realizar la practica a través un video o experimentalmente. } \\
\text { Relación entre el modelo de ID y la estrategia de RP, donde el } \\
\text { estudiante deberá a partir del problema planteado, generar } \\
\text { hipótesis que le permitirán dar solución al problema, y además, } \\
\text { indagar otra metodología que le permita resolver el problema } \\
\text { planteado. }\end{array}$ \\
\hline & 要 & 4 & $\begin{array}{l}\text { Investigación } \\
\text { dirigida (ID) }\end{array}$ & $\begin{array}{l}\text { El estudiante a partir de la observación de un video (reacción } \\
\text { química } \mathrm{HCl}+\mathrm{Zn} \text { ) deberá generar un problema y una solución } \\
\text { por medio de la indagación, generación de hipótesis, y la } \\
\text { experimentación, por tanto esta actividad se basa en la } \\
\text { estrategia de } \mathrm{ID} \text {. }\end{array}$ \\
\hline & 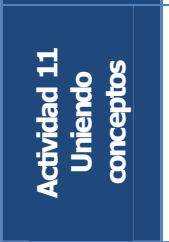 & 2 & Crucigrama & $\begin{array}{l}\text { Se presenta un crucigrama con diferentes preguntas sobre el } \\
\text { tema de reacciones químicas. } \\
\text { Esta Actividad es una estrategia que le permitirá al estudiante } \\
\text { poder consolidar los conocimientos que adquirió al desarrollar } \\
\text { todas las actividades realizadas para el tema de reacciones } \\
\text { químicas. }\end{array}$ \\
\hline
\end{tabular}


Metodología y diseño

El desarrollo de esta investigación descriptiva, se dividió en cinco fases, a saber: 1) identificación del problema, 2) Construcción del marco de referencia, 3) se establecen los conceptos a partir de los cuales se desarrolla el PGA, 4) Diseño del PGA y de los módulos para el docente y el estudiante; y 5) Organización y realización del trabajo escrito.

De esta forma, en la fase de diseño del Programa Guía De Actividades, "QUIMIVIDA: Cotidianidad y Ciencia", sobre dos temas fundamentales de la química, las mezclas y las reacciones químicas, se tuvo en cuenta la cotidianidad de los estudiantes, las situaciones que los rodean constantemente y que no son ajenas a ningún ser humano, por ejemplo, la oxidación de los metales, las mezclas presentes en bebidas alcohólicas, tintas, la comida, entre otros.

Se desarrollaron dos módulos dirigidos respectivamente a docente y a estudiante. El módulo docente, en el cual se presentan dos secciones, la inicial conceptualiza sobre los modelos utilizados y la otra presenta y describe las actividades, con algunos comentarios y/o sugerencias, para el docente, en el desarrollo de cada una de estas.

En el módulo estudiante, se presentan únicamente las actividades planteadas, con las siguientes características principales en su presentación: una sección denominada "Sabias que" en el inicio de cada actividad, como conceptualización del tema y ayuda conceptual; algunas ilustraciones con las aules se busca relacionar la cotidianidad del estudiante con el concepto a trabajar; y, en el caso del prácticas experimentales, se tienen en cuenta algunas preguntas básicas que ayudaran al estudiante a comprender el objetivo del laboratorio, al igual que se presenta una tabla donde se muestra la viabilidad de realizar o no la práctica en casa, además de la necesidad o no de utilizar elementos de protección como guantes, bata, entre otros.

Cabe resaltar, que en el diseño del PGA, también se utilizan herramientas didácticas como lo son: los mapas conceptuales, la V Heurística y las prácticas experimentales, las cuales aportan fuertemente al desarrollo de la evaluación, la cual es formativa, pues tiene en cuenta el proceso del estudiante y busca la retroalimentación constante.

\section{Conclusiones}

El diseño del Programa Guía de Actividades de química, que desarrolla actividades sobre los conceptos de mezdas y reacciones químicas, se realizó teniendo en cuenta el modelo de investigación dirigida y la estrategia de resolución de problemas.

Por otra parte, los conceptos a trabajar se establecieron a través de la revisión de los estándares curriculares de ciencias naturales, para los grados décimo y undécimo, y la relación de estos conceptos con el contexto del estudiante, conduyendo que los conceptos, mezdas y reacciones químicas, son fundamentales para la química y, por supuesto, para la vida del estudiante, quien puede aportar a la cultura y sociedad gracias a su proceso de formación escolar.

Al diseñar, entonces, un Programa Guía De Actividades basado en la Investigación Dirigida y en la estrategia de resolución de problemas, se logró profundizar en dicho modelo y en la estrategia, de tal forma que se aplicaron directamente a los conceptos de área ya establecidos.

Las actividades elaboradas para el PGA diseñado, podrán generar en los estudiantes de media vocacional, el mejoramiento en el desarrollo de competencias básicas de tipo científico escolar como lo son: argumentar, proponer e interpretar. 
Así, el desarrollo de competencias básicas en ciencias, puede ser posible a partir del uso y aplicación de modelos didácticos que muestren la relación entre la cotidianidad y el estudio en la escuela, por esto, en el PGA diseñado se busca la interacción constante entre el conocimiento científico y el cotidiano, llegando así al conocimiento científico escolar, a través del cual se muestra al estudiante una verdadera aplicación al conocimiento adquirido en la escuela.

Finalmente, al diseñar el PGA "QUIMIVIDA: Cotidianidad y Ciencia", se ponen al descubierto algunas de las principales características que se deben tener en cuenta para desarrollar un currículo de aula mucho más aplicado y que tiene en cuenta los modelos didácticos contemporáneos y la cotidianidad de los estudiantes, iniciando así un largo camino en el cual se diseñaran y aplicarán nuevas actividades para los diferentes temas tratados en la educación media, complementando el diseño inicial de dicho PGA, teniendo en cuenta los estándares curriculares, la coherencia, continuidad y contexto en que las actividades serán aplicadas.

\section{Bibliografía}

Hierrezuelo Moreno, J.; Molina González, E., y Yus Ramos, R. (1991): "Una nueva generación de materiales curriculares para la enseñanza de las ciencias: los programas guía de actividades", en Revista de Educación, No. 295, pp. 463-485.

Mora Zamora, Arabela. (2005). La Investigación dirigida. VII Congreso Nacional de Ciencias. Exploraciones dentro y fuera de aula. Costa Rica. 2005. Pág. 1.

Perales, F.J. La resolución de Problemas: (1993). Una Revisión Estructurada. Departamento de Didáctica de las ciencias Experimentales. Facultad de ciencias de la Educación. Universidad de Cartuja. Granada. Enseñanza de las Ciencias. (11 / 2). Pág. 170-178

http: //uww.seduca.gov.co/portal/herramientas/EstandaresCienciasSocialesCienciasNaturales. pdf Página de la Secretaría de educación de Colombia. 